\title{
Relationships between the kinetic, alternating-line, and Poggendorff illusions: The effects of interstimulus interval, inducing parallels, and fixation
}

\author{
PETER WENDEROTH and MICHAEL JOHNSON \\ University of Sydney, Sydney, Australia
}

\begin{abstract}
Recent reports have noted that dots that move obliquely upwards behind vertical parallels appear to emerge too high (kinetic illusion, or KI), and that alternating the left and right halves of the Poggendorff illusion increases alignment error. However, when the parallels are omitted, the KI remains large but the alternating line illusion is reduced markedly. In three experiments, this difference was not replicated: both successive effects were larger than static illusions, both increased with interstimulus interval, and introducing parallels added a constant to all three effects. Fixation versus pursuit made no difference. The data suggested that a simple eyemovement account of the KI was unlikely to be tenable. Deterioration of memory for spatial location of the first stimulus was considered a possible contributor.
\end{abstract}

When a moving spot is observed to pass orthogonally behind a rectangular mask and then to reemerge (Figure 1a), its velocity has to be increased while it is visually absent, in order to produce the perception of constant velocity, or smooth movement (Maruyama \& Iwasaki, 1973).

Nihei $(1973,1975)$ has shown that the moving-dot effect (originally called "the tunnel phenomenon"' by Maruyama \& Iwasaki, 1973) became an illusion of spatial distortion when the dot's motion path was oblique (Figure 1c). Now the dot appeared to emerge too high and even appeared to curve in as it approached the bar and to curve out on reemergence. Such apparent curvature of the oblique lines has been proposed by (for example) Wenderoth, Beh, and White (1978) as a factor in the Poggendorff illusion (Figure 1b). In addition, Nihei (1975) demonstrated that his "kinetic illusion" (KI) was larger with vertical parallels and an oblique motion path (Figure 1c) than in the reverse case (Figure 1d). This is an "oblique effect" (Appelle, 1972) which is also exhibited by the Poggendorff illusion (e.g., Green \& Hoyle, 1964).

The purpose of this study was to examine the KI and new variants of it, and to consider, in the light of the evidence, various explanations of the effect, including its possible relationship to the Poggendorff illusion.

This research was supported by the Australian Research Grants Committee, Grant A28015547 to the first author, which included the Research Assistantship of the second author. Other facilities were made available by a University of Sydney Research grant. The authors gratefully acknowledge all assistance. The authors' mailing address is: Department of Psychology, University of Sydney, Sydney, N.S.W. 2006, Australia.
The impetus for this work was provided by a recent rediscovery of the KI and reports of similar effects. Fineman and Melingonis (1977) not only rediscovered the KI, but added two new observations. First, the effect was reduced by only $25 \%$ when the parallels were absent so that there merely was a gap between a lower (upper) and upper (lower) motion path. That is, the moving dot simply disappeared in the middle segment of its oblique transverse (Figure 1e). This finding, if robust, demonstrates a clear difference between the KI and the Poggendorff illusion: the latter is reduced markedly in the parallelless version (Curthoys, Wenderoth, \& Harris, 1975; Day, 1973; Farné, 1970; Fineman \& Melingonis, 1977; Goldstein \& Weintraub, 1972).

Fineman and Melingonis (1977) also asked whether the KI could be due not to motion but simply to succession of the two oblique segments. To test this, they included two conditions, one with and one without parallels, in which one solid line transversal was on for $375 \mathrm{msec}$ and, after an interstimulus interval (ISI) of $750 \mathrm{msec}$, the second transversal appeared for $375 \mathrm{msec}$. Under these conditions, the apparent misalignment with parallels was slightly larger than the Poggendorff effect, but in the absence of parallels, the illusion was not larger than the static parallelless Poggendorff effect. Hence, they concluded, the large parallelless KI depended on motion, not merely on succession. The largest illusion of all was obtained for the KI with parallels (Figure 1c).

Another recent finding is that of Caelli and Dodwell (1980). They used a mathematical model to predict that the Poggendorff illusion would increase if the left-hand side of the figure (both the oblique 


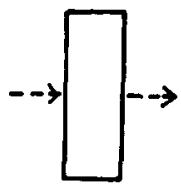

a

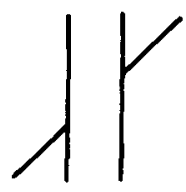

b

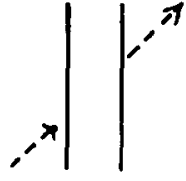

C

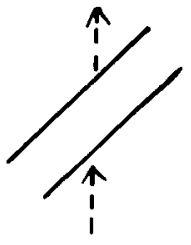

d

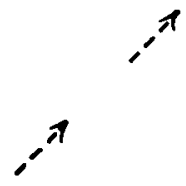

$\boldsymbol{\theta}$

Figure 1. Various lliusory phenomena, including (a) the "tunnel" phenomenon, (b) the Poggendorff illusion, (c, d) the kinetic illuoion (KI), and (e) the parallelless KI.

and the vertical) and the right-hand side were presented successively, in apparent motion, with an ISI of $20 \mathrm{msec}$. They obtained the predicted increase when apparent motion occurred and, curiously, stated that the increase would not occur with longer ISIs (e.g., $500 \mathrm{msec}$ ) without, however, testing this proposition. This is a questionable conclusion also, because Fineman and Melingonis's ISI was $750 \mathrm{msec}$, well beyond the limit for apparent motion. Perhaps the essential difference is that in one case the parallels were static and in the other they alternated together with the oblique. Alternatively, different factors might account for the effects obtained with longer ISIs.

One other aspect of the Caelli and Dodwell experiment is extremely puzzling. The illusions, given in degrees, averaged less than 1 deg in the static Poggendorff case and less than 2 deg in the alternating condition. The usual Poggendorff effect averages $6^{\circ}$ to $8^{\circ}$ (e.g., see below, Figure 3). Hence, Caelli and Dodwell (two of the three subjects) have either obtained an odd result or perhaps expressed the errors in terms not of angular subtense at the standardtransversal's tip-for which we may use the "oo" symbol-but in terms of angular subtense at the observer's eye (using “deg”). Unfortunately, one cannot check these possibilities, because no stimulus dimensions and procedures (viewing distances) are given.

The preceding review of the literature identifies so many variables that could be relevant to the magnitude of the KI and related effects that we viewed our first experiment as purely exploratory. Fineman and Melingonis (1977) strongly asserted that the KI was very likely due to eye movements that occur as the observer tracks the dot across the display. Their indirect evidence for this was Novak's (1966) finding that fixation results in a smaller Poggendorff illusion than does free inspection. This logic, of course, is doubly indirect because it also presumes an affinity between the Poggendorff and kinetic illusions. The eye-movement hypothesis is interesting, however, because Novak's is one of the few experiments to report reduction with fixation: Wenderoth, White, and
Beh (1978) failed to obtain any difference. Later, in Experiment 3, we reexamine this question.

\section{EXPERIMENT 1}

In Experiment 1, there were seven stimulus displays, designed to investigate a number of factors. The seven conditions, which were produced on a computer-linked visual display screen, are represented schematically in Figure 2. The aims of the experiment can best be described in relation to these stimulus conditions.

Condition 1 was the parallelless KI and Condition 2 was the same, except that after the 500 -msec ISI, instead of the right oblique track, a dot, which remained stationary for $1.33 \mathrm{sec}$, appeared where the KI track would have begun. (The time constant of $1.33 \mathrm{sec}$ was chosen because this is the time the $\mathrm{KI}$ dot requires to traverse its $42.4-\mathrm{mm}$ track at a velocity of $3.19 \mathrm{~cm} \mathrm{sec}^{-1}$.) In Condition 3, the lefthand track proceeded in the opposite direction and then the dot of Condition 2 appeared. Condition 4 differed from Condition 2 only in that as the dot moved, it traced a line that remained on until the dot had traversed its path. The same was true of Condition 5, except that the dot direction was reversed.

The last two conditions examined conditions of alternation rather than motion, one with a line and dot (Condition 6) and one with a pair of lines (Condition 7).

It was intended that these conditions would investigate numerous aspects of the KI. First, did the KI require an extended right-hand stimulus (Condition 1 vs. Condition 2) and how did that variable affect the alternating stimuli (6 vs. 7)? Second, if pursuit eye movements were relevant, perhaps large differences would occur with opposite dot directions ( 2 vs. $3 ; 4$ vs. 5 ). We also wished to reexamine the difference between the KI and alternating lines ( 1 vs. 7). Finally, because the previous comparison (Fineman \& Melingonis, 1977) between the KI and alternating lines did not indicate whether the KI was so much larger because of motion or because of the absence of a complete left line, Conditions 4 and 5 rectified 
1

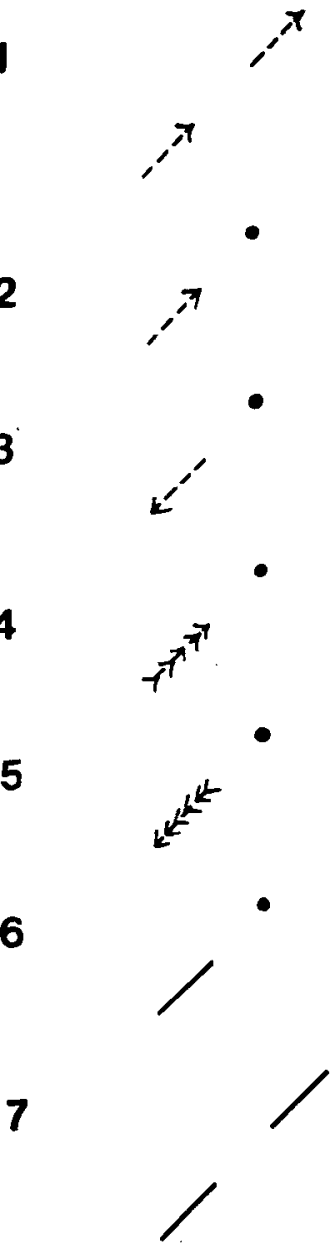

Figure 2. The seven display conditions of Experiment 1. See text for explanation and rationale.

this omission by including both a moving dot and a left line. Note that no parallels were used in this experiment. Also, we used mainly dot rather than extended right-hand stimuli because preliminary informal observation had suggested roughly equivalent effects with dots or extended paths.

\section{Method}

Apparatns. Stimuli were presented on the flat face of a Tektronix 608 display (P4 phosphor), interfaced with a HewlettPackard 1350A Graphics Translator and a PDP-11/20 computer. With head maintained still, upright, and centered by means of a padded chinrest, subjects viewed the screen in a totally dark room, following 5 min dark adaptation, from a distance of $57 \mathrm{~cm}$. The display screen was masked by a black circular cardboard mask, $100 \mathrm{~mm}$ in diameter. All of the oblique lines or paths were $42.4 \mathrm{~mm}$ long and oriented $45^{\circ}$ to vertical. The vertical parallels used in Experiment 3 were $100 \mathrm{~mm}$ in length. The oblique distance between lines or dots in which no stimulus appeared was $28.2 \mathrm{~mm}$. Dots moved at a velocity of $3.19 \mathrm{~cm} \mathrm{sec}^{-1}$. These dimensions resemble those used by Fineman and Melingonis (1977), although our ISI of $500 \mathrm{msec}$ was less than their $750 \mathrm{msec}$ ISI. The contrast of lines at different orientations was visibly different at low lu- minances. Hence, Polaroid sheets were mounted over the screen, the luminance was increased, until the effective luminance of all lines was about $3 \mathrm{~cd} \mathrm{~m}^{-2}$ and contrast of all lines was about 1.0 (because the background luminance was too low to measure). The subjects judged whether the variable stimulus appeared to be too high or too low by pressing an upper or lower microswitch, respectively.

Procedure. The subjects were permitted to inspect the stimuli freely; no fixation instructions were given. Each subject completed each of the seven conditions in a different random order. For each condition, the PSE was determined by a double, randomly interleaved staircase (Cornsweet, 1962), each staircase beginning at a randomly chosen position within $10 \mathrm{~mm}$ of true alignment.

When 3 reversals in each staircase had occurred with a 3-mm step size, the latter was reduced to $1 \mathrm{~mm}$ for 10 more reversals, 5 in each staircase. The PSE was based on the mean of these last 10 reversals.

Subjects. There were 16 subjects, all volunteers from introductory courses in psychology. Those who wore corrective lenses did so in the experiment.

\section{Results}

The mean alignment errors, in both millimeters and degrees, are shown for each of the seven conditions in Table 1 , along with standard errors. Variability was relatively high in all cases, as it was in the Fineman and Melingonis (1977) study, because subjects found the task difficult. All errors were positive (right-hand track, point or line set too low). The mean angular error was calculated from the formula $45-\tan ^{-1}(20-x) / 20$, where $x$ is the mean misalignment, in millimeters.

Statistically, the data were subjected to a simple subjects $\times$ treatments analysis of variance (Winer, 1962, chap. 4), and six planned contrasts were tested. Although the obtained mean KI (Condition 1) appeared slightly larger than the means obtained when the right-hand stimulus was a dot (Conditions 2 to 5), the $\mathrm{KI}, 6.25^{\circ}$, was not significantly different from the mean of these four other conditions, $4.94^{\circ}$, with $F(1,84)>.05$.

Within the latter four conditions, the direction of dot movement (Conditions 2 vs. 3 and 4 vs. 5) made no difference, with $F(1,84)=0.06$ and 0.44 , respectively. Furthermore, whether the dot merely moved

Table 1

Alignment Errors (in Millimeters and Degrees) and Standard Errors (in Millimeters) Obtained in Conditions 1 to 7, Experiment 1

\begin{tabular}{cccc} 
& \multicolumn{2}{c}{ ME } & SE \\
\cline { 2 - 3 } Condition & $\mathrm{mm}$ & $\mathrm{deg}$ & $\mathrm{mm}$ \\
\hline 1 & 3.95 & 6.25 & 0.85 \\
2 & 3.07 & 4.75 & 0.88 \\
3 & 3.34 & 5.21 & 1.48 \\
4 & 3.53 & 5.53 & 0.94 \\
5 & 2.77 & 4.25 & 1.09 \\
6 & 1.33 & 1.97 & 0.98 \\
7 & 4.56 & 7.34 & 1.02 \\
\hline
\end{tabular}

Note $-M E=$ mean error $; S E=$ standard error $; m m=$ millimeters; deg $=$ degrees. 
or whether it traced a line (Conditions $2+3$ minus Conditions $4+5)$ was irrelevant $[F(1,84)=0.73$, $\mathrm{p}>$.05].

However, when static stimuli alternated (Conditions 6 and 7), the alternating lines produced a large effect $\left(7.34^{\circ}\right)$, significantly greater than that which occurred with alternating line and dot $\left(1.97^{\circ}\right)$, with $F(1,84)=7.96, p<.01$. Because the line-line effect was so large, the mean of the two alternating conditions, $4.66^{\circ}$, was not significantly different from the mean of the other five motion conditions, $5.20^{\circ}$, with $F(1,84)=0.33$. The large line-dot versus lineline difference currently is under further study, but was not explored further in these experiments.

\section{Discussion}

The most unexpected result was the very large illusion that occurred with alternating lines (Condition 7). In this case, Fineman and Melingonis (1977) obtained a minimal effect, more like what we obtained in Condition 6. Therefore, and because we decided to check on this finding for the two main reasons outlined below, we turn to Experiment 2 before discussing the implications of all the Experiment 1 data.

\section{EXPERIMENT 2}

If large, robust alignment errors do occur with alternating lines, then, for reasons unknown, Fineman and Melingonis obtained an aberrant result, and motion, rather than succession, is not necessary to induce large errors. One aim of Experiment 2 was to determine whether large alternating-line illusions occurred at a variety of ISIs, spanning the ISI used by Fineman and Melingonis. In addition, Caelli and Dodwell (1980) alternated Poggendorff halves only: they did not use a parallelless version. We were unable to establish, from their paper, whether their model predicted enhanced illusions in the absence of parallels, but, at any rate, they claimed (in the absence of data) that no enhancement would occur with ISIs outside the apparent motion range (around $20 \mathrm{msec}$ ). ${ }^{1}$ For this reason, and also because the ISI in our Experiment 1 was $500 \mathrm{msec}$, we varied ISI in Experiment 2 and included a 20-msec ISI.

Experiment 2 included two stimulus configurations only, the KI (Condition 1, Experiment 1) and alternating lines (Condition 7, Experiment 1). However, in each case, four values of ISI were examined: 20 , 320,620 , and $920 \mathrm{msec}$. Thus, the ISI range spanned apparent motion ( $20 \mathrm{msec}$ ), our Experiment 1 value (500 msec), Fineman and Melingonis's ISI (750 msec), and a very long ISI that unequivocally resulted in perceived succession rather than motion $(920 \mathrm{msec})$.

\section{Method}

All methods and procedures were as in Experiment 1. Thirteen volunteers from introductory courses were the subjects.

\section{Results}

The mean alignment errors, in millimeters and degrees, together with standard errors, are given in Table 2. The data were analyzed as in Experiment 1, using seven planned contrasts.

For the dot (KI) conditions, linear trend was significant across ISI $[F(1,84)=4.83, p<.05]$. Quadratic $(F=1.24)$ and cubic $(F=0.20)$ trends were not. In the case of alternating lines, none of the linear $(F=2.23)$, quadratic $(F=0.001)$, or cubic $(F=0.06)$ trends was significant, although the means appeared to increase with ISI and the largest trend component was linear. Overall, there was no significant difference between the mean $\mathrm{KI}\left(8.78^{\circ}\right)$ and the mean alternating-line illusion $\left(7.54^{\circ}\right)$.

\section{Discussion}

Taken together, Experiments 1 and 2 seem to demonstrate convincingly that both a large $\mathrm{KI}$ and a large alternating-line illusion occur in the absence of inducing parallels, contrary to the finding of Fineman and Melingonis (1977), who obtained only a large KI under such conditions. Furthermore, we found, in Experiment 2, that the KI increases linearly with ISI and that the alternating line effect certainly does not decrease with ISI. Since Caelli and Dodwell claimed that they would find an enhanced Poggendorff effect only when the ISI was one that produced the smallest of our nevertheless large effects, it seems possible that the similarity between the effects studied here, and their illusion, is minimal. Again, perhaps this relates to their use of alternating obliques and parallels.

Experiment 1, Conditions 2 to 5, provided some extremely indirect, but suggestive, evidence against Fineman and Melingonis's eye-movement hypothesis to explain the KI. While those conditions in which the moving dot traced right to left (Conditions 3 and 5) need not necessarily have induced eye movements quantitatively or qualitatively different from those in which motion was left to right (Conditions 2 and 4), if eye-movement patterns were the explanation it is at least a little surprising that these conditions were not different.

\begin{tabular}{|c|c|c|c|c|c|c|}
\hline \multirow[b]{3}{*}{ ISI } & \multicolumn{3}{|c|}{$\mathbf{K I}$} & \multicolumn{3}{|c|}{ Alternating Lines } \\
\hline & \multicolumn{2}{|c|}{ ME } & \multirow{2}{*}{$\frac{\mathrm{SE}}{\mathrm{mm}}$} & \multicolumn{2}{|c|}{ ME } & \multirow{2}{*}{$\frac{\mathrm{SE}}{\mathrm{mm}}$} \\
\hline & $\mathrm{mm}$ & $\operatorname{deg}$ & & $\mathrm{mm}$ & deg & \\
\hline 20 & 4.60 & 7.40 & 0.75 & 4.13 & 6.58 & 0.91 \\
\hline 320 & 4.13 & 6.57 & 0.62 & 4.34 & 6.94 & 0.84 \\
\hline 620 & 4.43 & 9.12 & 0.75 & 5.02 & 8.16 & 0.74 \\
\hline 920 & 7.03 & 12.03 & 1.21 & 5.19 & 8.48 & 1.47 \\
\hline
\end{tabular}

Note-ME = mean error $: S E=$ standard error $; m m=$ millimeters; deg $=$ degrees. 
However, to test more directly the hypothesis that pursuit eye movements are not necessary for the KI, subjects in Experiment 3 were instructed sometimes to pursue the stimulus and sometimes to fixate throughout.

\section{EXPERIMENT 3}

The main aim of this experiment was to compare not only the static Poggendorff illusion, the KI, and the alternating line effect under pursuit and fixation conditions, but also the three effects with and without parallels. The logic behind the latter aim relates to the tenability of various kinds of theories of the Poggendorff and related illusions. Some theories assert that similar mechanisms are involved in the parallelless and complete figures. Eye-movement theories and theories that stress the perceptual elongation of vertical space are examples. Other theories claim independence of the two effects by stressing the key role of the parallels in producing the effects-parallel attraction is one example-and, indeed, Quina-Holland (1977) concludes that the parallelless Poggendorff does not account for any significant proportion of the variance of her measures taken on the full figure (albeit length measures rather than misalignment errors). Also, theories stressing lines bending or bowing at intersects seem not able to explain the small, but significant, parallelless effect.

A question of interest, therefore, is whether the addition of parallels to the KI, the alternating-line illusion, and the static Poggendorff illusion will increase each effect by the same amount, or whether the larger effects will be increased more, resulting in a significant interaction between the nature of the test stimulus and the presence or absence of parallels.

\section{Method}

General methods and procedures were unchanged. However, in all conditions, the stimulus display was preceded by a small cross located at the point where the left oblique would meet the parallel (or where the left motion path would end, the left oblique would end, or ended, etc.). This cross appeared $500 \mathrm{msec}$ prior to the presentation of the stimulus display in all 12 conditions: static, KI, alternating lines $(3) \times$ parallels, no parallels $(2) \times$ pursuit, fixation (2).

Each subject completed the 12 conditions in a different random order. Prior to each trial, the word SCAN or STARE appeared on the screen for $\mathbf{5 0 0} \mathrm{msec}$ to indicate a pursuit or fixation trial, respectively; after an off time of $200 \mathrm{msec}$, the cross appeared; $500 \mathrm{msec}$ later, the left-hand stimulus began and, after a further $1.33 \mathrm{sec}$, the cross and the left-hand stimulus both disappeared. Note that if the cross had remained present throughout the trial the task could have been performed by simply aligning the righthand stimulus and the fixation point. Under STARE conditions, the subject was instructed to fixate the cross or the point where the cross had been throughout the trial; under SCAN conditions, the cross was ignored, and moving (pursuit) or alternating (saccade) stimuli were visually followed.

At the beginning of each condition, the SCAN or STARE instruction appeared on the screen for $1.5 \mathrm{sec}$, and the briefer, 500-msec SCAN or STARE instruction served as a reminder prior to individual trials within a condition.

The subjects were 21 undergraduate volunteers, 10 from an advanced course and 11 from introductory courses, but all were totally naive regarding the aims and expectations of the experimenters.

The ISI was always $750 \mathrm{msec}$, the same as that used by Fineman and Melingonis.

\section{Results}

The mean alignment errors and standard errors, in millimeters, are shown in Table 3, and the errors, in degrees, in Figure 3.

Once again, a simple analysis of variance with 11 planned contrasts characterized the data as follows. In the absence of parallels, pursuit versus fixation made no difference to the static Poggendorff illusion $[F(1,220)=0.73]$, the $\mathrm{KI}(\mathrm{F}=0.15)$, or the alternatingline effect $(F=3.82)$, with $p>.05$ in each case. There were also no such differences in the with-parallels effect, the respective $F$ values being $0.58,0.29$, and 0.36 , again with $\mathrm{p}>.05$.

The obvious linear trend, from small Poggendorff to large alternating-line effects, was significant $(F=$ $24.58, p<.001)$; quadratic trend was not $(F=0.35$, $p>.05)$. There was no significant interaction between the with- and without parallels functions, either under pursuit $(F=0.27, p>.05)$ or under fixation $(F=1.67, p>.05)$ conditions. Finally, the overall with-parallels error $\left(9.20^{\circ}\right)$ was significantly larger than the parallelless effect $\left(3.42^{\circ}\right)$.

\section{GENERAL DISCUSSION}

The first point to note is that we have obtained, in Experiment 3 , the usual $1^{\circ}-2^{\circ}$ static parallelless

Table 3

Alignment Errors (in Millimeters) for the Poggendorff, Kinetic, and Alternating Line Illusions as a Function of Presence or Absence of Parallels and Fixation (Stare) or Pursuit (Scan) Instructions, Experiment 3

\begin{tabular}{llccc}
\hline & & \multicolumn{3}{c}{ Effect } \\
\cline { 3 - 5 } & & $\begin{array}{c}\text { Static } \\
\text { Poggendorff }\end{array}$ & Kinetic & $\begin{array}{c}\text { Alternating } \\
\text { Lines }\end{array}$ \\
\hline \multirow{4}{*}{ Scan } & M & 1.11 & Without Parallels \\
& SE & 0.30 & 0.98 & 0.94 \\
Stare & M & 1.41 & 2.27 & 2.22 \\
& SE & 0.34 & 0.89 & 0.76 \\
& & & With Parallels \\
Scan & M & 4.77 & 5.23 & 6.83 \\
& SE & 0.40 & 0.91 & 0.78 \\
Stare & M & 4.17 & 5.89 & 6.41 \\
& SE & 0.60 & 0.95 & 0.65 \\
\hline
\end{tabular}




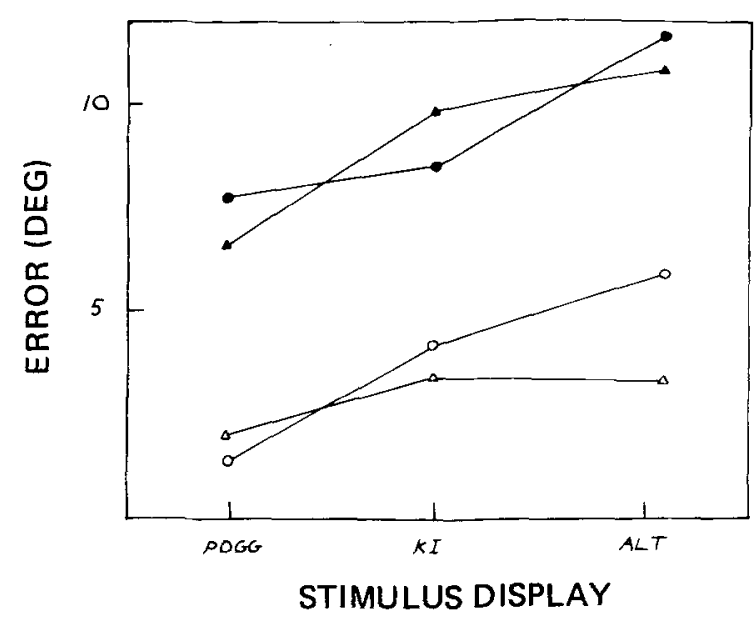

Figure 3. Mean alignment errors, Experiment 3, obtained with fixation (triangles) and without fixation (circles) and with parallels (filled symbols) and without parallels (open symbols) for the Poggendorf, kinetic, and alternating-line illusions.

Poggendorff effect and also the usual $6^{\circ}-8^{\circ}$ static, full effect. This result perhaps verifies, in some sense, the rest of the results. Also, as we have shown previously (Wenderoth, White, \& Beh, 1978), free inspection versus oblique tip fixation makes no difference to the Poggendorff illusion, contrary to Novak's (1966) result.

Indeed, since fixation or pursuit in no way affected any of the three illusions, it now seems extremely unlikely that eye movements account for any of the effects, with or without parallels.

Once again, the alternating line illusion was large, both with and without parallels and, in terms of the obtained linear trend, exceeded even the KI. We have no explanation of the fact that Fineman and Melingonis failed to obtain a large without-parallels effect with the same ISI.

Perhaps the most interesting result is the lack of interaction between illusion type and parallel presence or absence: the addition of parallels seems to add a constant amount of error to each of the three illusions, the actual mean increases being $5.41^{\circ}, 5.35^{\circ}$, and $6.59^{\circ}$ for the Poggendorff, $\mathrm{KI}$, and alternating lines, respectively. This suggests that whatever the factors are that account for the differences between the three illusions, they are not differentially affected by the presence of parallels. In turn, this seems to suggest that the explanation of the data in Figure 3 will require one (or more) mechanisms to explain the effect of the parallels; and one (or more) different mechanisms to explain the differences between the three efffects. In this context, we note that in the KI with parallels, the dot was observed by us and other subjects to curve in perceptually as it approached the parallel. Curiously, this was apparent only on approach, not exit, whether the dot moved left to right or right to left (down). However, no hint of such bowing was seen in the parallelless case. The difficulty with the idea that the effect of the parallels is line- or dot-path bowing unfortunately is that despite indirect evidence for this (e.g., Wenderoth, White, \& Beh, 1978), the obliques in the Poggendorff figure, and other related displays, visually do not appear bent, and direct measures do not reveal such bending (e.g., Wenderoth, Parkinson, \& White, 1979).

Another, more general, implication of the finding of linear trend with no interaction-that the parallels add a constant to each illusion-is that the control procedures usually used in Poggendorff studies are justified. That is, it is usual to subtract parallelless (control) errors from with-parallel effects (experimental) to measure the effect of the parallels. The data in Figure 3 appear to justify this procedure.

As to the explanation of the KI without parallels, one hypothesis follows from the fact that we obtained a large alternating-line illusion as well, and that both of these parallelless effects increased with ISI in Experiment 2. The proposal is simply that misalignment is overestimated or underestimated (see below) as a function of temporal delay between the misaligned targets, whether this is a true perceptual phenomenon or some sort of cognitive memory related error. It could be that distance between any two successive stimuli is misjudged; this could be tested by devising means of estimating the separation between successive points, as a function of ISI, experiments we plan to conduct. It is not clear whether over- or underestimation is predicted: the upper right KI path could appear too high either by its shifting perceptually to the left (underestimation of gap) or by its shifting vertically up (overestimation). Experiments in which the judged oblique distance (judged gap) was measured could differentiate these two possibilities.

The one remaining result which is not consistent with this notion is the very small alternating line-dot illusion obtained in Experiment 1, Condition 6. We intend to investigate this case further, to determine whether this result is robust. The reason for some suspicion is that the KI effects with dots (Experiment 1 , Conditions 2 to 5 ) were relatively large. On the other hand, there is independent evidence that the line-dot static parallelless effect is smaller than the line-line effect (Curthoys et al., 1975). ${ }^{2}$

Additional research also is required into the Caelli and Dodwell (1980) claim that alternating the left and right halves of the Poggendorff illusion magnifies the effect only with ISIs producing apparent motion. This needs to be tested directly, because our largest effects occurred out of that ISI range and Caelli and Dodwell made the claim in the absence of data. Finally, we are aware of the fact that Matin, Pola, Matin, and Picoult (1981) obtained constant 
errors in vernier alignment with sequentially flashed targets when subjects tried to maintain prior fixation. They attributed these errors to involuntary eye movements and deterioration of memory for spatial location of the first stimulus. Since their effects also increased with ISI, the same two factors might have contributed to our results. Fixation instructions do not necessarily eradicate eye movements, and we would like to measure eye movements directly in future experiments.

Additional experiments have been conducted to test the hypothesis that the $\mathrm{KI}$ and its variants are attributable, in part at least, to attraction between the parallel lines when they are present. We prefer to desist from further theoretical speculation until both the present data and those being analyzed (Johnson \& Wenderoth, Note 1) have been reported.

\section{REFERENCE NOTE}

1. Johnson, M., \& Wenderoth, P. The kinetic illusion and the nature of its explanation. Manuscript in preparation, 1983.

\section{REFERENCES}

Appelle, S. Perception and discrimination as a function of stimulus orientation: The 'oblique effect' in man and animals. Psy. chological Bulletin, 1972, 78, 266-278.

Caelli, T., \& Dodweld, P. On the contours of apparent motion: A new perspective on visual space-time. Biological Cybernetics, $1980,39,27-35$.

CoRnsweet, T. The staircase method in psychophysics. American Journal of Psychology, 1962, 75, 485-491.

Curthoys, I., Wenderoth, P., \& Harris, J. The effects of motion path and the length of the variable segment in the Poggendorff illusion without parallels. Perception \& Psychophysics, 1975, 17, 358-362.

DAY, R. H. The oblique line illusion: The Poggendorff effect without parallels. Quarterly Journal of Experimental Psychology, 1973, 25, 535-541.

FaRNE, M. On the Poggendorff illusion: A note to Cumming's criticism of Chung Chiang's theory. Perception \& Psychophysics, 1970, 8, 112.

Fineman, M., \& Melingonis, M. The effect of a moving dot transversal on the Poggendorff illusion. Perception \& Psychophysics, 1977, 21, 153-156.

Goldstein, M., \& Weintraub, D. J. The parallel-less Poggen- dorff: Virtual contours put the illusion down but not out. Perception \& Psychophysics, 1972, 11, 353-355.

Green, R. T., \& Hoyle, E. M. The influence of spatial orientation on the Poggendorff illusion. Acta Psychologia, 1964, 22, 348-366.

Maruyama, K., \& Iwasaki, S. "Prospective perception" and the tunnel phenomenon. Tohoku Psychologica Folia, 1973, 32, 132-144.

Matin, L., Pola, J., Matin, E., \& Picoult, E. Vernier discrimination with sequentially-flashed lines: Roles of eye movements, retinal offsets and short term memory. Vision Research, 1981, 21, 647-656.

NiHe I, Y. A preliminary study on the geometrical illusion of motion path: The kinetic illusion. Tohoku Psychologica Folia, $1973,32,108-114$.

NiHE1, Y. The effect of direction of motion on the magnitude of the geometrical illusion of motion path: The kinetic illusion (II). Tohoku Psychologica Folia, 1975, 34, 88-94.

Novak, S. Effects of free inspection and fixation on the Poggendorff illusion. Perceptual and Motor Skills, 1966, 23, 663-670.

Quina-Holland, K. Spatial distortions within the Poggendorff illusion and its variants: A parametric analysis. Perception \& Psychophysics, 1977, 21, 118-124.

Wenderoth, P., BEH, H., \& White, D. Perceptual distortion of an oblique line in the presence of an abutting vertical line. Vision Research, 1978, 18, 923-930.

Wenderoth, P., Parkinson, A., \& White, D. A comparison of visual tilt illusions measured by the techniques of vertical setting, parallel matching and dot alignment. Perception, 1979, 8, 47-57.

WENDE Roth, P., White, D., \& BEH, H. The effect of peripheral and central fixation on a Poggendorff-like vernier alignment task. Perception \& Psychophysics, 1978, 24, 377-386.

Winer, B. J. Statistical principles in experimental design. New York: McGraw-Hill, 1962.

\section{NOTES}

1. The Caelli and Dodwell model seems to predict enhanced illusions with alternation, because the direction of scan path changes when the oblique intersects the vertical, resulting in a perceived upwardly curved motion path between the two apparent motion stimuli. If this is correct, they should not predict large parallelless effects because the static parallelless illusion, with no intersects presents, is itself very small.

2. We have replicated the result. In another experiment, under similar conditions, we obtained a KI of $3.06 \mathrm{~mm}$ and a line-dot effect of $0.97 \mathrm{~mm}$, and the latter effect was comparable to a static, parallelless Poggendorff effect of $1.04 \mathrm{~mm}$.

(Manuscript received December 27, 1982; revision accepted for publication April 22, 1983.) 